\title{
Liquid Computing in a Simplified Model of Cortical Layer IV: Learning to Balance a Ball
}

\author{
Dimitri Probst ${ }^{1,3}$, Wolfgang Maass ${ }^{2}$, Henry Markram ${ }^{1}$ \\ and Marc-Oliver Gewaltig ${ }^{1}$ \\ 1 Blue Brain Project, École Polytechnique Fédérale de Lausanne, \\ CH-1015 Lausanne, Switzerland \\ marc-oliver.gewaltig@epfl.ch \\ 2 Institute for Theoretical Computer Science, Technische Universität Graz, \\ A-8010 Graz, Austria \\ 3 Ruprecht-Karls Universität Heidelberg, D-69117 Heidelberg, Germany
}

\begin{abstract}
We present a biologically inspired recurrent network of spiking neurons and a learning rule that enables the network to balance a ball on a flat circular arena and to steer it towards a target field, by controlling the inclination angles of the arena. The neural controller is a recurrent network of adaptive exponential integrate and fire neurons configured and connected to match properties of cortical layer IV. The network is used as a liquid state machine with four action cells as readout neurons. The solution of the task requires the controller to take its own reaction time into account by anticipating the future state of the controlled system. We demonstrate that the cortical network can robustly learn this task using a supervised learning rule that penalizes the error on the force applied to the arena.
\end{abstract}

Keywords: Spiking neural networks, NEST, dynamic control task, neurobotics, brain-inspired computing, AdEx, supervised learning, closedloop motor control.

\section{Introduction}

Controlling a physical dynamical system with a realistic spiking neuronal circuit in a closed loop is difficult for several reasons. First, the intrinsic time constants of the physical system and those of the neurons may differ greatly. Second, the neural controller must implicitly learn the kinematics of the dynamical system. And third, in closed perception-action loops, small errors of the controller may amplify to the point where the system can no longer be controlled. Joshi and Maass [1] used a liquid-state machine [4 based on a general microcircuit model and linear readout units to control the movements of a robot arm. In an alternative approach, Bouganis and Shanahan $[3]$ used spike-timing dependent plasticity (STDP) to teach a spiking network how to control a robot arm with four degrees of freedom. In this work, we trained a cortex-like network to solve a dynamic control task. 
Dynamic control tasks, like pole-balancing or balancing a ball on a moving surface, are particularly challenging because the system continues to evolve while the controller determines its next action and by the time the controller applies its force, it may no longer be appropriate. Thus, such tasks can only be solved if the controller takes its own reaction time into account by anticipating the state of the system when the control force is actually applied.

In this paper, we investigate if a recurrent network of spiking neurons, mimicking a piece of layer IV of a rat somatosensory cortex, can be trained to steer a rolling ball towards a target by controlling the inclination angles of the surface that the ball is rolling on. Following earlier work of Maass et al. [4, we use liquid computing to solve this task. In this paradigm, the recurrent network is used to map a spatio-temporal stimulus into a high-dimensional space. The desired response or target function is then constructed by readout neurons or action cells which compute a weighted sum of the high-dimensional activity in the recurrent network. In our case, there are four action cells, controlling the forces which can change the inclination angles of the arena.

\section{Control Task and Network Architecture}

The system to be controlled consists of a ball with mass $m_{\text {ball }}$ and radius $r_{\text {ball }}$ that rolls on a flat circular arena with a reflecting border at radius $r_{\text {arena }}$ (Figure 1). The inclination of the arena is determined by two orthogonal angles $\alpha$ and $\beta$. The ball can roll on the arena and is subject to two forces, gravity and rolling resistance. If the arena is tilted against the horizontal plane, gravity will

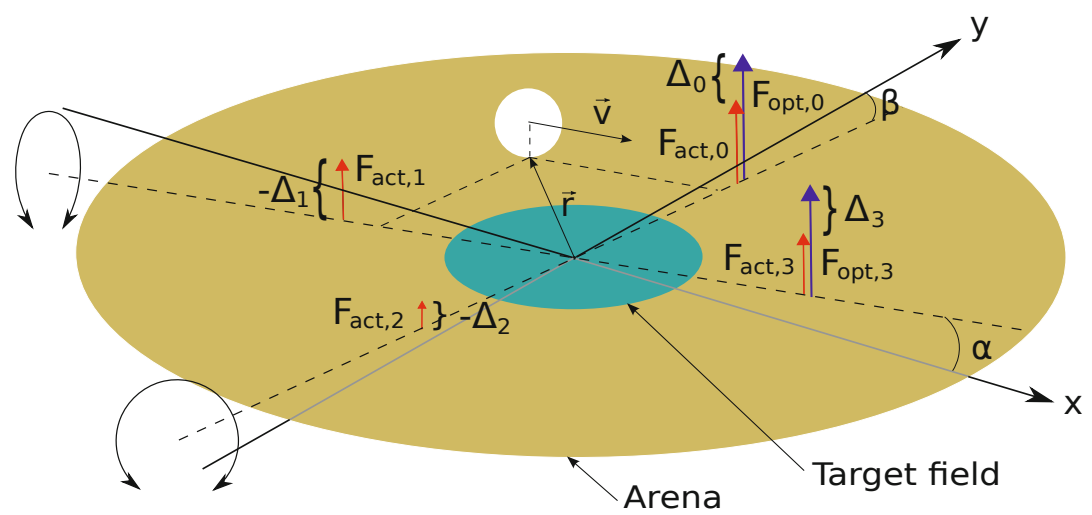

Fig. 1. Illustration of the control task: A ball (white) of mass $m_{\text {ball }}$ moves on a circular arena (yellow) which can be rotated around two orthogonal axes. The goal of the task is to bring the ball into the target field (blue) by tilting the arena. In order to change the inclination angles, the forces $F_{\text {act }, 0}$ to $F_{\text {act,3 }}$ are applied which compose $\boldsymbol{F}_{\text {act }}$. Depending on the current position $\boldsymbol{r}$ and velocity $\boldsymbol{v}$, an optimal force $\boldsymbol{F}_{\text {opt }}$ is computed according to Equation 3 . Here, the components $F_{\text {opt,1 }}$ and $F_{\text {opt,2 }}$ are zero. The differences $\Delta_{0}$ to $\Delta_{3}$ are used for learning. Note, sizes are not to scale. 
accelerate the ball, thereby increasing its kinetic energy. If the arena is aligned with the horizontal plane, rolling resistance will be maximal and the kinetic energy will dissipate until the ball comes to rest. The strength of this dissipative force is determined by the coefficient of rolling friction $\eta$ and the coefficient of restitution $\rho$.

The controller should solve the following task. Starting from an arbitrary initial position and velocity (within appropriate ranges) and given the current position of the ball, the controller must apply the appropriate forces to steer the ball into the target field at the center of the arena.

The forces are applied at four force points $F_{\text {act }, 0}, F_{\text {act }, 1}, F_{\text {act, } 2}$, and $F_{\text {act,3 }}$ (cf. Figure 1). Each angle is controlled by two opposing forces, similar to antagonistic muscle pairs.

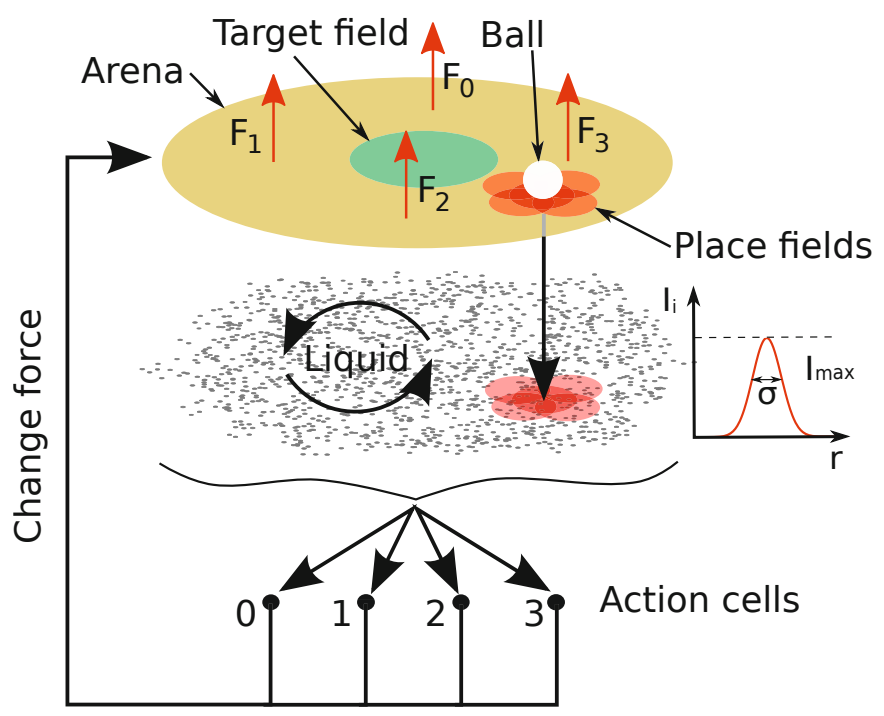

Fig. 2. Illustration of the closed-loop motor control: Neurons in the liquid (grey dots) receive position-dependent Gaussian activation currents from within their place fields (red). The neurons are randomly distributed in a hexagon which is topologically mapped onto the arena. The resulting spiking activity reverberates in the liquid through recurrent connections. Four action-cells integrate a weighted sum of the activity in the liquid. Finally, the membrane potential of the action cells is transformed to a motor force which changes the inclination of the arena. Drawing not to scale.

As neural controller we use a recurrent network of adaptive exponential integrate and fire neurons [5] and four non-spiking action cells, each representing a population of motor neurons. The properties of the neurons and their connections are chosen to mimic the properties of layer IV of a rat somatosensory cortex. In the circuit, neurons are randomly positioned in three dimensions with an exponential connection probability that depends on the distance between the 
neurons (see Table 22). To read the position of the ball, the excitatory neurons have place fields on the surface of the arena. The locations and distribution of the place fields are determined by logically stretching the two horizontal dimensions of the circuit to the size of the arena (see Figure 2). If the ball is near the place field of a neuron $i$, the neuron receives a current of the form:

$$
I_{i}(\boldsymbol{r})=I_{\max } \cdot \exp \left(-\frac{\left(\boldsymbol{r}_{\boldsymbol{i}}-\boldsymbol{r}\right)^{2}}{2 \sigma^{2}}\right) .
$$

Here, $I_{\max }$ denotes the maximum current, $\sigma$ the size of a place field and $\boldsymbol{r}_{\boldsymbol{i}}$ the position of the centre of the $i$ th place field [8]. The response of the controller is generated by four action cells which integrate the activity of the excitatory neurons in the circuit. From the membrane potential $V_{\mathrm{m}, j}$ of each action cell $j$ we compute the force $F_{j}$ according to:

$$
F_{j}\left(V_{\mathrm{m}, j}\right)=\frac{F_{\max }}{1+\exp \left(-2 \cdot\left(V_{\mathrm{m}, j}+70 \mathrm{mV}\right)\right)} .
$$

The details of the model are summarized in Table 2. The dynamics of ball and arena were simulated in Python. The neural controller was simulated with the Neural Simulation Tool NEST [6] .

\section{Learning Paradigm and Experimental Results}

To train the controller, we use a supervised learning algorithm that penalizes the difference between the optimal force $\boldsymbol{F}_{\mathbf{o p t}}$ and the actually applied force. To avoid oscillations, the control must take the reaction time into account. Thus, given the position of the ball at time $t$, the network has to compute the required force at a later time $t+t_{\mathrm{f}}$.

The force is split into the four components $F_{\mathrm{opt}, 0}$ to $F_{\mathrm{opt}, 3}$ in the following way: Only those two force components are non-zero which are adjacent to the quadrant in which the ball will reside at time $t+t_{\mathrm{f}}$ :

$$
\left\{\begin{array}{l}
\left(F_{\text {opt }, 1} \text { or } F_{\text {opt }, 3}\right)=F_{\text {abs }} \cdot \cos \phi \\
\left(F_{\text {opt }, 0} \text { or } F_{\text {opt }, 2}\right)=F_{\text {abs }} \cdot \sin \phi
\end{array}\right.
$$

with the absolute value of the force

$$
F_{\text {abs }}=F_{\max } \cdot\left(\frac{1}{1+\exp \left(-16 \cdot\left(\left|\boldsymbol{r}_{\text {guess }}\right|-0.16 \mathrm{~m}\right)\right)}-\frac{1}{15}\right) .
$$

The vector $\boldsymbol{r}_{\text {guess }}$ and the value $\phi$ are specified as

$$
\boldsymbol{r}_{\text {guess }}\left(t_{\mathrm{f}}\right)=\boldsymbol{r}+\boldsymbol{v} \cdot t_{\mathrm{f}}+\frac{1}{2} \cdot \boldsymbol{a} \cdot t_{\mathrm{f}}^{2},
$$

and

$$
\phi=\arctan \left|\frac{y_{\text {guess }}}{x_{\text {guess }}}\right| .
$$


Here, $\boldsymbol{a}$ is the acceleration of the ball and $F_{\max }$ the force maximum. $x_{\text {guess }}$ and $y_{\text {guess }}$ are the components of the vector $\boldsymbol{r}_{\text {guess }}$. The force in Equation 4 will be larger than the weight force of the ball, if the ball is outside its target field, and it will match the weight force, if the ball is inside the target field.

We applied the learning algorithm 1 to adjust the weights between the recurrent network (the liquid) and the action cells.

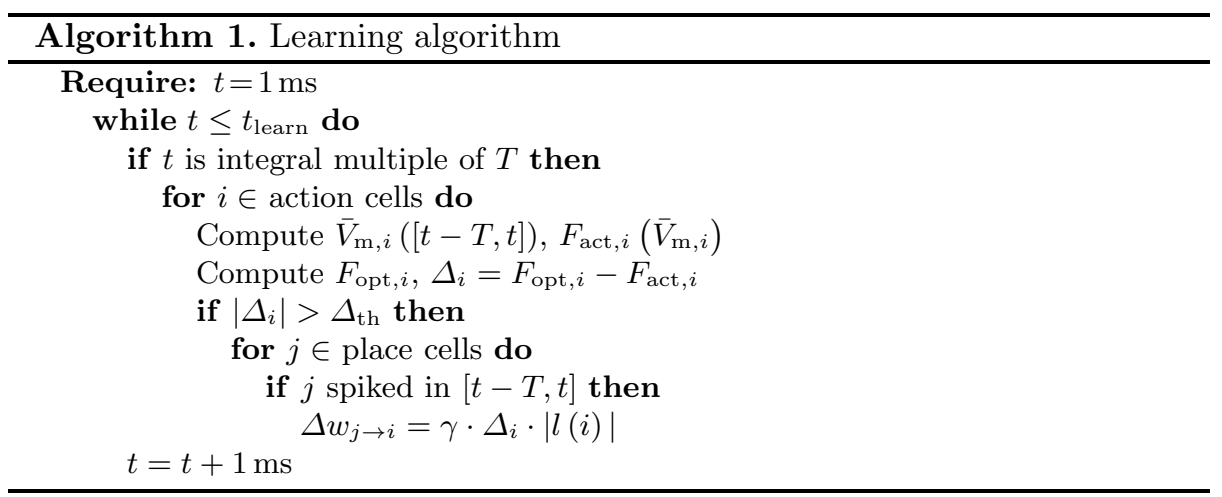

Here, $T$ is the learning period and $\Delta_{\text {th }}$ is the maximum tolerable error. $\bar{V}_{\mathrm{m}, i}([t-T, t])$ is the mean potential of action cell $i$ over the latest period T. $w_{j \rightarrow i}$ denotes the conductance of the connection from neuron $j$ to action cell $i$. The coefficient $\gamma$ is the learning rate, which we kept constant. The factor $l(i)$ corresponds to the y-coordinate of the position of the ball if $i$ is even, else to the x-coordinate. Near the target field, $l(i)$ provides a small weight adjustment. Otherwise, it reduces the time when the ball stays near the border of the arena. Table 1 summarizes the values of the task variables and the learning parameters.

Table 1. Task and learning parameters

\begin{tabular}{lcccr}
\hline Name & Value Name & Value Name & Value Name & Value \\
\hline \hline$F_{\max }[\mathrm{N}]$ & $7.2 \alpha_{\max } / \beta_{\max }\left[{ }^{\circ}\right]$ & $5.0 t_{\mathrm{f}}[\mathrm{ms}]$ & $200.0 I_{\max }[\mathrm{pA}]$ & 500.0 \\
\hline$m_{\text {ball }}[\mathrm{g}]$ & $264.0 r_{\text {arena }}[\mathrm{cm}]$ & $50.0 T[\mathrm{~ms}]$ & $20.0 \sigma[\mathrm{cm}]$ & 2.5 \\
\hline$r_{\text {force }}[\mathrm{cm}]$ & $25.0 \eta$ & $0.08 \gamma\left[\frac{\mathrm{nS}}{\mathrm{Nm}}\right]$ & $1.0 t_{\text {learn }}[\mathrm{s}]$ & 10.0 \\
\hline$r_{\text {ball }}[\mathrm{cm}]$ & $2.0 \rho$ & $0.6 \Delta_{\text {th }}[\mathrm{N}]$ & $0.1 t_{\text {test }}[\mathrm{s}]$ & 20.0 \\
\hline
\end{tabular}

Each experiment is divided into training runs of duration $t_{\text {learn }}$ and test runs of duration $t_{\text {test }}$. At the beginning of a run (training and testing), the ball is placed at a random position $\boldsymbol{r}_{0}$ with a random velocity $\boldsymbol{v}_{0}$ lower than $v=0.3 \frac{\mathrm{m}}{\mathrm{s}}$. After that, the trajectory of the ball is determined by the dynamics of the arena and the forces $F_{\text {act, } 0}$ to $F_{\text {act,3 }}$ applied by the network. There are five learning runs for each test run. 
Figure 3 shows the results after 2500 seconds of learning, when no further improvement of the performance could be observed. Panel A shows the root mean square error for each component of the applied force. Each point denotes the result of a test run after the respective time of training. From $t=350 \mathrm{~s}$ on, the the error signal reaches the value $\bar{\Delta}_{i}=(0.27 \pm 0.11) \mathrm{N}$, which is sufficient to steer the ball into the target field.

High errors between $t=1000 \mathrm{~s}$ and $t=2000 \mathrm{~s}$ in Figure 3A occur if the test run starts at a position which has not been trained before. Figure $3 \mathrm{~B}$ shows the final position of the ball in a test run as a function of the learning time. Except for the failed runs, the ball stays close to the target field $\left(\bar{r}_{\text {end }}=(6.7 \pm 2.8) \mathrm{cm}\right)$.
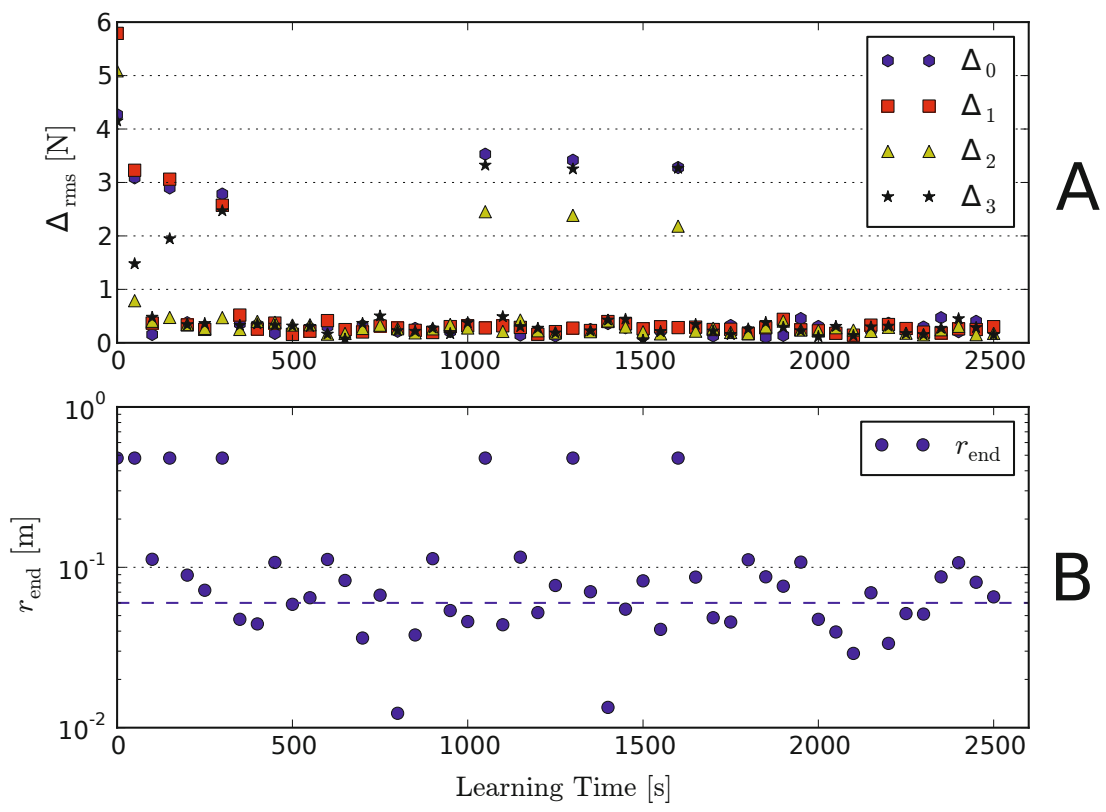

Fig. 3. Results of a learning session. Each measurement denotes the result of a test run after the indicated learning time. (A) The root mean squared error for each force component $\Delta_{i}$ as a function of the learning time. (B) Final position of the ball in a test trial as a function of the learning time. The blue dashed line shows the border of the target field. 
Table 2. Model description according to [7]. Errors are given as standard deviations.

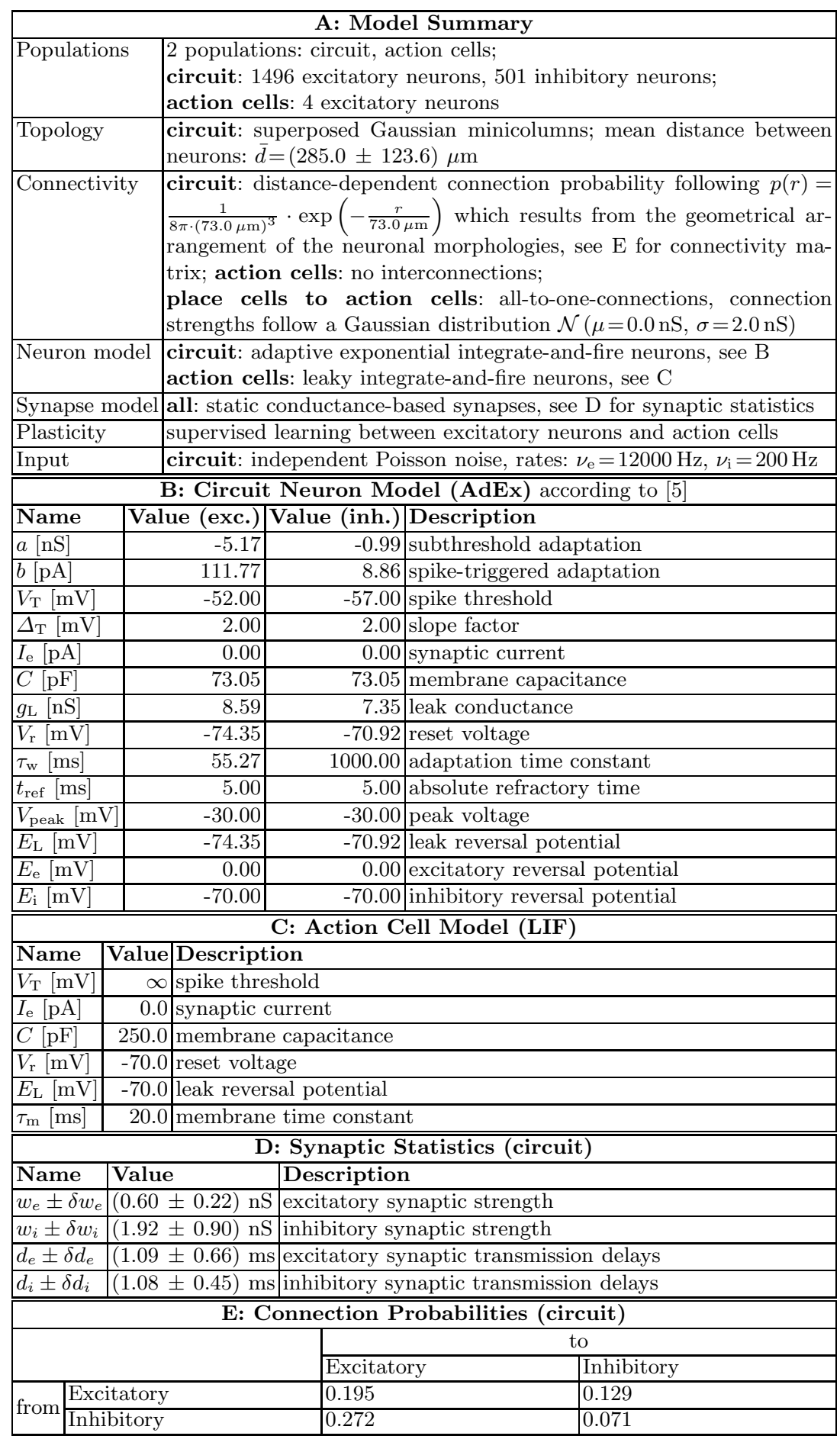




\section{Discussion}

In this paper, we presented a neural controller based on spiking neurons and liquid computing that solves a dynamic control task. Using a supervised learning rule that penalizes the error between the applied force and the required force, we adapt the weights to the four action cells. The input to the controller encodes the position of the ball at time $t$. The output is the force vector that controls the table. After learning, the system manages to keep the ball within or very close to the target field. Since the force output is produced with some delay $t_{\mathrm{f}}$, the performance is limited by the ability of the network to predict the position and velocity of the ball at the time when the force is applied.

Another important aspect of the system is that the time scale of the control task and the time scales of the controller must be compatible. The time scale of the controller is determined by the time constants of neurons and the connection delays, which are determined by biological processes and are beyond our control. The time scale of the control task mainly depends on the maximal inclination angle of the arena and the friction between the arena and the ball.

Acknowledgements. Partial funding was provided from the European Union project FP7-269921 (BrainScaleS).

\section{References}

1. Joshi, P., Maass, W.: Movement Generation with Circuits of Spiking Neurons. Neural Computation 17, 1715-1738 (2005)

2. Burgsteiner, H.: Training Networks of Biological Realistic Spiking Neurons for RealTime Robot Control (2005)

3. Bouganis, A., Shanahan, M.: Training a Spiking Neural Network to Control a 4DoF Robotic Arm based on Spike Timing-Dependent Plasticity. In: IEEE World Congress on Computational Intelligence, Barcelona, pp. 4104-4111 (2010)

4. Maass, W., Natschlaeger, T., Markram, H.: Real-Time Computing Without Stable States: A New Framework for Neural Computation Based on Perturbations. Neural Computation 14(11), 2531-2560 (2002)

5. Brette, R., Gerstner, W.: Adaptive Exponential Integrate-and-Fire Model as an Effective Description of Neuronal Activity. J. Neurophysiol. 94, 3637-3642 (2005)

6. Gewaltig, M.-O., Diesmann, M.: NEST (Neural Simulation Tool). Scholarpedia 2(4), $1430(2007)$

7. Nordlie, E., Gewaltig, M.-O., Plesser, H.E.: Towards Reproducible Descriptions of Neuronal Network Models. PLoS Comput. Biol. 5(8), e1000456 (2009)

8. Vasilaki, E., Frémaux, N., Urbanczik, R., Senn, W., Gerstner, W.: Spike-Based Reinforcement Learning in Continuous State and Action Space: When Policy Gradient Methods Fail. PLoS Compuatational Biology 5(12), e1000586 (2009) 